\title{
Spectrum and Eigenfunctions of a Differential Operator Arising by Linearization of the Fisher and Related Equations
}

\author{
E. J. M. VELING* \\ Mathematical Centre, Amsterdam, The Netherlands
}

[Received 5 May 1983]

\begin{abstract}
For a class of semilinear diffusion problems from population genetics the linearized differential equation is studied in order to estimate the rate of exponential convergence to some stable stationary solution. Some monotonicity properties of the lowest eigenvalue with respect to the parameters of the problem are given. Two types of lower bounds for this eigenvalue are constructed and compared. For the Fisher non-linearity it turns out that the eigenvalue problem can be solved by an explicit representation of the eigenfunction as a hypergeometric polynomial. For the cubic non-linearity the eigenfunction can be represented by a Heun function.
\end{abstract}

\section{Introduction}

MANY PROBLEMS from population genetics are described by semilinear parabolic differential equations. If, for example, one studies the frequency $u(x, t)$ of an allele $\mathrm{A}$ in a diploid population with zygotes AA, Aa and aa, where the carriers of the alleles are restricted to a half-bounded one-dimensional habitat, then one encounters the problem

$$
\left.\begin{array}{rl}
u_{t}=u_{x x}+f(u), \quad & (x, t) \in Q=\mathbb{R}^{+} \times \mathbb{R}^{+}, \\
u(x, 0)= & g(x), \quad x \geqslant 0, \\
u(0, t)=h(t), \quad t \geqslant 0,
\end{array}\right\}
$$

where $f(u)$ represents some non-linearity depending on the relative fitnesses of the homozygotes AA and aa with respect to the heterozygote Aa. The following classes of non-linearities $f \in \mathscr{F}=\mathscr{F}_{1} \cup \mathscr{F}_{2}$ will be treated:

$$
\begin{aligned}
\mathscr{F}_{1}= & \left\{f \mid f \in C^{3}([0,1]), f(0)=f(1)=0, f^{\prime}(0)<0, f^{\prime}(1)<0,\right. \text { there exists a } \\
& \text { number } a, 0<a<1, \text { such that } f(u)<0 \text { on }(0, a) \text { and } f(u)>0 \text { on } \\
& \left.(a, 1), \int_{0}^{1} f(u) d u>0\right\}, \\
\mathscr{F}_{2}= & \left\{f \mid f \in C^{3}([0,1]), \quad f(0)=f(1)=0, \quad \begin{array}{r}
f^{\prime}(0)>0, \\
\left.f^{\prime}(1)<0, \quad f(u)>0 \text { on }(0,1)\right\} .
\end{array}\right.
\end{aligned}
$$

* Present address: Rijksinstituut voor Drinkwatervoorziening (R.I.D.), Postbus 150, $2260 \mathrm{AD}$ Leidschendam. The Netherlands. The main part of the research was performed when the author was a member of the Department of Applied Mathematics of the Mathematical Centre, Amsterdam, The Netherlands. 
The class $\mathscr{F}_{1}$ represents the so-called heterozygote inferior case, where the zygote AA is the most viable, and the class $\mathscr{F}_{2}$ represents the heterozygote intermediate case, where again AA is the most viable genotype. Characteristic examples are

$$
\begin{gathered}
f_{a}(u)=u(1-u)(u-a), \quad 0<a<\frac{1}{2}, \quad f_{a} \in \mathscr{F}_{1}, \\
\tilde{f}_{v}(u)=u(1-u)(1+v u), \quad v>-1, \quad \tilde{f}_{v} \in \mathscr{F}_{2} .
\end{gathered}
$$

$\mathscr{F}_{2}$ is known as the class of the Fisher type non-linearity with characteristic representative $\tilde{f}_{0}$ (Fisher, 1937).

If one specifies the initial and boundary conditions as follows

$$
\left.\begin{array}{l}
h(t) \text { is non-decreasing, } t \geqslant 0, \lim _{t \rightarrow \infty} h(t)=\theta, \theta \in[0,1], \\
\qquad g(x)= \begin{cases}q(x), & x \in(a, b), \\
0, & x \in \overline{\mathbb{R}^{+}} \backslash(a, b),\end{cases} \\
\text { with } a>0, \quad q(a)=q(b), \quad q^{\prime \prime}+f(q)=0,
\end{array}\right\}
$$

then it is known (Aronson \& Weinberger, 1975, Proposition 5.1) that $\lim _{t \rightarrow \infty} u(x, t)=V(x)$, uniformly on bounded sets. $V(x)$ satisfies

From the expression

$$
\left.\begin{array}{c}
V^{\prime \prime}+f(V)=0, \quad x>0, \quad{ }^{\prime}=\frac{d}{d x}, \\
V(0)=\theta, \quad V(\infty)=1, \quad \theta \in[0,1] .
\end{array}\right\}
$$

with

$$
\frac{1}{2}\left(V^{\prime}\right)^{2}+F(V)=F(1), \quad x \geqslant 0,
$$

$$
F(u)=\int_{0}^{u} f(v) d v
$$

and from the properties of $f \in \mathscr{F}$ it follows that $V(x)$ is a strictly increasing function with the asymptotic behaviour

$$
1-V(x)=C e^{-b x}[1+o(1)], \quad x \rightarrow \infty, \quad b=\sqrt{-f^{\prime}(1)},
$$

for some positive constant $C$. We label this function as $V_{\theta}$. For the study of this stationary solution by means of the principle of linearized stability it is necessary to consider in the Hilbert space $\mathscr{L}^{2}(0, \infty)$ the eigenvalue problem defined by $(1.10)$ and (1.11)

$$
\begin{gathered}
N[w] \equiv-w^{\prime \prime}-f^{\prime}\left(V_{\theta}(x)\right) w=\lambda w, \quad x>0, \\
w(0)=0,
\end{gathered}
$$

where $N[w]$ is obtained by linearizing (1.7) around the stationary solution $V_{\theta}(x)$. In this paper we pay attention to this eigenvalue problem.

The type of parabolic equations as (1.1) allows travelling wave solutions $u(x, t)=U(z)$, where $z=x-c t, c \in \mathbb{R}$ and $U(z)$ satisfies

$$
\left.\begin{array}{rl}
U^{\prime \prime}+c U^{\prime}+f(U) & =0, \quad z \in \mathbb{R}, \quad '=\frac{d}{d z}, \\
U(-\infty) & =1, \quad U(\infty)=0 .
\end{array}\right\}
$$


For $f \in \mathscr{F}_{1}$ there exists a unique positive number $c_{0}$ which depends on $f$ and for $f \in \mathscr{F}_{2}$ there exists a half-line $[c(f), \infty)$ of possible velocities, such that for all $c \geqslant c(f)$ there exists a solution $U_{c}(z)$ of (1.12). See Aronson \& Weinberger (1975), Fife (1979) and Fife \& McLeod (1977) for more biological background, a detailed derivation of Equation (1.1) and more mathematical results, mostly concerning the corresponding Cauchy problem. The last paper treats the stability of the travelling wave. In Veling $(1981,1982 a)$ problem (1.1) was considered for a broad class of initial and boundary conditions and it was proved that if $h(t)$ tends exponentially to a limit $\theta, \theta \in[0,1]$, for $t \rightarrow \infty$, the solution converges for $x \in \overline{\mathbb{R}^{+}}$to an asymptotic state which consists of a travelling wave $U$ and the solution $V_{\theta}$. At this study interest arose in the eigenvalue problem (1.10), (1.11) as an independent problem.

In Section 2 we study the spectrum of the self-adjoint operator A associated with $N$ and it is proved that $\sigma(A) \subset(0, \infty)$. Some additional information about $\sigma(A)$ is gathered in Section 2. For this operator $A$ there may exist points in the point spectrum: in that case $m=\inf \{\lambda \mid \lambda \in \sigma(A)\}$ is an isolated point of $\sigma(A)$ and is denoted by $\lambda_{1}$. To emphasize the dependence of $\lambda_{1}$ on the parameter $\theta$ and the nonlinearity $f$ we shall write also $\lambda(\theta, f)=\lambda_{1}$ where we suppress the index 1 .

In Section 3 some monotonicity properties are proved with respect to the parameter $\theta$ for fixed $f$ and with respect to different functions $f$ for fixed $\theta$, namely

$$
\begin{aligned}
& \lambda\left(\theta_{1}, f\right) \geqslant \lambda\left(\theta_{2}, f\right), \quad \text { if } \quad \theta_{1} \geqslant \theta_{2}, \\
& \lambda\left(\theta, f_{1}\right) \geqslant \lambda\left(\theta, f_{2}\right), \quad \text { if } \quad f_{1}^{\prime}(u)<f_{2}^{\prime}(u), f_{1}^{\prime \prime}(u) \leqslant 0 \quad \text { on } \quad[\theta, 1] \text {. }
\end{aligned}
$$

In Section 4 we shall obtain the following lower bounds for $\lambda_{1}$.

$$
\begin{gathered}
\lambda(\theta, f) \geqslant f^{2}(\theta) /\{2[F(1)-F(\theta)]\}, \quad \text { if } \quad f^{\prime \prime}(u) \leqslant 0 \quad \text { on }[\theta, 1], \\
\lambda(\theta, f) \geqslant-f^{\prime}(1)-c_{p}\|q\|_{p}^{(2 p) /(2 p-1)}, \quad p \geqslant 1,
\end{gathered}
$$

with

and

$$
\|q\|_{p}^{p}=\int_{0}^{\infty}|q|^{p} d x
$$

$$
\begin{gathered}
c_{1}=\frac{1}{4}, \quad c_{p}=p^{-(2 p) /(2 p-1)}(p-1)^{(2 p-2) /(2 p-1)}\left[\frac{\Gamma\left(\frac{1}{2}\right) \Gamma(p)}{\Gamma\left(p+\frac{1}{2}\right)}\right]^{-2 /(2 p-1)}, p>1,(1.17) \\
q(x)=f^{\prime}(1)-f^{\prime}\left(V_{\theta}(x)\right) .
\end{gathered}
$$

The condition $f^{\prime \prime} \leqslant 0$ on $[\theta, 1]$ we needed for $(1.15)$ can be relaxed somewhat.

In Section 5 we solve the eigenvalue problem for the Fisher non-linearity $f_{0}(u)=u(1-u)$ explicitly by means of a quadratic transformation. It turns out that the eigenfunction can be written as a hypergeometric polynomial. The results of Sections 2 and 3 are illustrated by this example.

In Section 6 we prove that the eigenvalue problem for the cubic $f_{a}(u)=u(1-u)(u-a)$ can be reduced to finding a zero for a Heun function. This knowledge can be used to calculate the eigenvalue numerically.

In Section 7 numerical results are presented with respect to the calculation of the eigenvalue for $f_{a}(u)$ by means of the method of Section 6 and by a finite-element method. In this section we also compare the constructed lower bounds for this 
calculated eigenvalue. It turns out that the bound (1.16) is superior to (1.15) as far as this example is concerned. It is possible to apply (1.16) to other problems of estimating the lowest eigenvalue from below. It applies to eigenvalue problems with a point spectrum and a continuous spectrum.

\section{The Spectrum $\sigma(A)$}

We consider the eigenvalue problem (1.10), (1.11). In order to be consistent with the usual setting for singular Sturm-Liouville problems we define the differential expression $M$

$$
M[w] \equiv-w^{\prime \prime}+q(x) w, \quad x>0, \quad q(x)=f^{\prime}(1)-f^{\prime}\left(V_{\theta}(x)\right) .
$$

The coefficient $q$ is real-valued and by $(1.9) q \in \mathscr{L}^{p}(0, \infty)$ for all $p \geqslant 1$ (even for $p>0$ ). By means of the following definition of $\mathscr{D}(T)$ we introduce the operator $T$ (see Naimark, 1968; Everitt, 1972):

$$
\begin{gathered}
\mathscr{D}(T)=\left\{w \mid w \in \mathscr{L}^{2}(0, \infty), w^{\prime} \text { absolutely continuous on }[0, X] \text { for all } X>0,\right. \\
\left.w(0)=0, \quad M[w] \in \mathscr{L}^{2}(0, \infty)\right\}, \\
T w=M[w], \quad w \in \mathscr{D}(T) .
\end{gathered}
$$

Next we define the operator $A$ as

$$
A w=N[w], \quad w \in \mathscr{D}(A)=\mathscr{D}(T),
$$

so this implies the identity $A=T-f^{\prime}(1)$. Further, there exists a $1-1$ correspondence between $\sigma(A)$ in the sense that

$$
\lambda \in \sigma(A) \Leftrightarrow \lambda+f^{\prime}(1) \in \sigma(T) .
$$

So all information about $\sigma(T)$ is easily translated into that for $\sigma(A)$. We introduce the following subsets of the complex plane $\mathbb{C}$, where $R_{\mu}=(T-\mu I)^{-1}$ and $E_{\mu}$ is the linear manifold spanned by the eigenvectors for $\mu$,

$$
\begin{gathered}
P \sigma(T)=\left\{\mu \mid \mu \in \mathbb{C}, R_{\mu}\right. \text { is a bounded operator defined on the } \\
\text { whole of } \left.\mathscr{L}^{2}(0, \infty) \ominus E_{\mu}\right\}, \\
C \sigma(T)=\left\{\mu \mid \mu \in \mathbb{C}, R_{\mu}\right. \text { is an unbounded operator defined on a } \\
\text { set which is dense in } \left.\mathscr{L}^{2}(0, \infty)\right\}, \\
P C \sigma(T)=\left\{\mu \mid \mu \in \mathbb{C}, R_{\mu}\right. \text { is an unbounded operator defined on a } \\
\text { set which is dense in } \left.\mathscr{L}^{2}(0, \infty) \ominus E_{\mu}\right\},
\end{gathered}
$$

(see Chaudhuri \& Everitt, 1967). Now we formulate

THEOREM 2.1 Let the operator $T$ be defined by (2.1), (2.2), (2.3). Let $f \in \mathscr{F}$, then the spectrum $\sigma(T)$ can be decomposed as

with the properties

$$
\sigma(T)=P \sigma(T) \cup C \sigma(T) \cup P C \sigma(T)
$$

(i) $(-\infty, 0) \cap P \sigma(T)$ is finite (possibly empty),

(ii) $P C \sigma(T)=\emptyset$,

(iii) $\quad E \sigma(T) \equiv C \sigma(T) \cup P C \sigma(T)=C \sigma(T)=[0, \infty)$. 
Proof. See Naimark $[(1968, \S 24.2$, Theorem 5 and Example a)]. The fact that $q$ as defined in $(2.1)$ is element of $\mathscr{L}^{1}(0, \infty)$ by (1.9) is sufficient for the proof. $C \sigma(T)=[0, \infty)$ implies $C \sigma(A)=\left[-f^{\prime}(1), \infty\right)$.

The following two lemmas supply information as to whether or not the set $\operatorname{P\sigma }(T)$ is empty.

Lemma 2.1 If there exists a real-valued function $\rho \in C^{2}([0, \infty))$ with the properties

(i) $\rho V_{\theta}^{\prime} \in \mathscr{D}(T)$,

(ii) $\chi_{1} \equiv\left\|\rho^{\prime} V_{\theta}^{\prime}\right\|_{2}^{2} /\left\|\rho V_{\theta}^{\prime}\right\|_{2}^{2}<-f^{\prime}(1)$,

then \# $\{\mu \mid \mu \in P \sigma(T)\}(=\#\{\lambda \mid \lambda \in P \sigma(A)\}) \geqslant 1$.

Proof. Since $\rho V_{\theta}^{\prime} \in \mathscr{D}(T)$ and $V_{\theta}^{\prime}(0) \neq 0$ we need $\rho(0)=0$. The lowest eigenvalue $\mu_{1}$ or the infimum of $C \sigma(T)$ (if $P \sigma(T)$ is empty) can be characterized by

$$
\mu_{1}=\inf _{\psi \in \mathscr{A}(T)}(\psi, M[\psi]) /(\psi, \psi) .
$$

If for some choice $\psi \in \mathscr{D}(T)(\psi, M[\psi]) /(\psi, \psi)<0$, then $\mu_{1}<0$, and so there exists at least one point in the set $\operatorname{P\sigma }(T)$. Making the choice $\psi=\rho V_{\theta}^{\prime}$ a calculation of $\left(\psi,-\psi^{\prime \prime}\right)$ reveals by partial integration

$$
\left(\psi,-\psi^{\prime \prime}\right)=\int_{0}^{\infty}\left(\rho^{\prime}\right)^{2}\left(V_{\theta}^{\prime}\right)^{2} d x-\int_{0}^{\infty} \rho^{2} V_{\theta}^{\prime} V_{\theta}^{\prime \prime \prime} d x,
$$

and so, since by (1.7) $V_{\theta}^{\prime \prime \prime}+f^{\prime}\left(V_{\theta}\right) V_{\theta}^{\prime}=0$, we find

$$
(\psi, M[\psi])=\left\|\rho^{\prime} V_{\theta}^{\prime}\right\|_{2}^{2}+f^{\prime}(1)\left\|\rho V_{\theta}^{\prime}\right\|_{2}^{2} .
$$

This means that by (ii) $(\psi, M[\psi]) /(\psi, \psi)<0$, and thus $\mu_{1}<0$.

In Section 7 results will be presented for some numerical calculations for the choice $f=f_{a} \in \mathscr{F}_{1}$ and $\rho=e^{\gamma x}-1, \gamma>0$.

LEMMA 2.2 If one of the following conditions has been satisfied

(i) $d_{p} \int_{0}^{\infty} x^{2 p-1}|q(x)|^{p} d x<1$, for some $p \geqslant 1$,

$$
\text { with } d_{1}=1, \quad d_{p}=(p-1)^{p-1} \Gamma(2 p) /\left\{p^{p}(\Gamma(p))^{2}\right\}, \quad p>1,
$$

(ii) $\frac{2}{\pi} \int_{0}^{\infty}|q(x)|^{\frac{1}{2}} d x<1, q<0, q$ monotonely increasing,

then $P \sigma(T)=\emptyset$.

Proof. See Dunford \& Schwartz (1963, Ch. 13, §9, H12) for (i), $p=1$ or Reed \& Simon (1978, Theorem XIII.9) for (i) and (ii).

In Section 5 condition (ii) will be used for $f=\widetilde{f_{0}} \in \mathscr{F}_{2}$. In Section 7 results will be presented for some numerical calculations for $f=f_{a} \in \mathscr{F}_{1}$.

The following information can be given about $\sigma(A)$ when $P \sigma(A) \neq \emptyset$. Let us introduce the hypotheses

$$
\exists \lambda_{1} \in \operatorname{P} \sigma(A),
$$

$\exists \mu_{1} \in P \sigma(T)$, 
which means that there exists an eigenvalue $l \in \mathscr{D}(A)=\mathscr{D}(T)$ such that $A l=\lambda_{1} l$ and $T l=\mu_{1} l$.

THEOREM 2.2 Let $(H \lambda)[$ or $(H \mu)]$ be satisfied, then

(i) $\lambda_{1}\left(\right.$ or $\left.\mu_{1}\right)$ is a simple eigenvalue,

(ii) $l(x)>0, \quad x>0$,

(iii) $l \in B C^{2}([0, \infty))$,

(iv) $\lambda_{1}>0\left[\right.$ or $\left.\mu_{1}>f^{\prime}(1)\right]$.

Proof. For (i) and (ii) we refer to Titchmarsh (1962, Ch. 5, §4) or Dunford \& Schwartz (1963, Ch. 13, $\$ 7$, Theorem 55) and for (iv) to Veling (1981, 1982a). Property (iii) follows from the fact that $f^{\prime}(u)$ is bounded on $[0,1]$, so $l^{\prime \prime} \in \mathscr{L}^{2}(0, \infty)$. Since $f^{\prime}\left(V_{\theta}(x)\right)$ is continuously differentiable, it follows by standard theory that $l$ is two times continuously differentiable on $(0, \infty)$. Using an interpolation lemma in Adams (1975, Ch. 4.10) we find that also $l^{\prime} \in \mathscr{L}^{2}(0, \infty)$. By a well-known embedding theorem there holds $l \in B C^{1}((0, \infty))$ and by $\left.N[l]=\lambda_{1}, f \in C^{3}([0,1)]\right)$ also $l^{\prime \prime} \in B C^{1}((0, \infty))$. Together with $l(0)=0$ this gives finally $l \in B C^{2}([0, \infty))$. Property (iv) implies $\sigma(A) \subset(0, \infty)$ as was announced in the Introduction.

In the next two sections the following lemma will be used repeatedly.

Lemma 2.3 Let $(H \lambda)$ be satisfied. Suppose there exists a function $w \in B C^{2}([0, \infty))$, $w(x)>0$ on $[0, \infty)$ and a positive number $\delta$ such that

$$
N[w]=-w^{\prime \prime}-f^{\prime}\left(V_{\theta}(x)\right) w \geqslant \delta w, \quad x>0,
$$

then the lowest eigenvalue $\lambda_{1} \in P \sigma(A)$ satisfies $\lambda_{1} \geqslant \delta$.

Proof. From Protter \& Weinberger $(1966,1967)$ it is known that

$$
\lambda_{1} \geqslant \inf (N[w(x)] / w(x) \mid 0<x<\infty)
$$

from which the statement of the lemma follows easily.

\section{Monotonicity of the First Eigenvalue}

Throughout this section we shall assume that $(H \lambda)$ is satisfied. Two monotonicity properties of the first eigenvalue are proved: the first (Theorem 3.1) with respect to the parameter $\theta$, the second (Theorem 3.2) with respect to the non-linearity $f$. Let $\lambda\left(\theta_{i}, f_{i}\right)$, $l^{\theta_{i}, f_{i}}$ denote respectively the first eigenvalue and eigenfunction for the operator $A^{\theta_{i}, f_{i}}$. In the sequel the indices are suppressed if there is no cause for confusion.

THEOREM 3.1 Let $(H \lambda)$ be satisfied both for $A=A^{\theta_{1}}, A^{\theta_{2}}$, then the following inequality holds

$$
\lambda\left(\theta_{1}, f\right) \geqslant \lambda\left(\theta_{2}, f\right), \text { if } \quad \theta_{1}>\theta_{2} .
$$

Proof. Define the positive number $\bar{x}$ as the shift such that

$$
V_{\theta_{2}}(\bar{x})=V_{\theta_{1}}(0)=\theta_{1} \text {. }
$$

Since $V_{\theta}$ is given by (1.8), it follows that $V_{\theta_{2}}(x+\bar{x})=V_{\theta_{1}}(x), x \geqslant 0$. Define $w(x)=l^{\theta_{2}}(x+\bar{x})$, then 
which is identical with

$$
\left\{\begin{array}{c}
-w^{\prime \prime}-f^{\prime}\left(V_{\theta_{2}}(x+\bar{x})\right) w=\lambda\left(\theta_{2}, f\right) w, \quad x>0 \\
w(x)=l^{\theta_{2}}(x+\bar{x})>0, \quad x \geqslant 0
\end{array}\right.
$$

$$
\left\{\begin{array}{c}
-w^{\prime \prime}-f^{\prime}\left(V_{\theta_{1}}(x)\right) w=\lambda\left(\theta_{2}, f\right) w, \quad x>0 \\
w(x)>0, \quad x \geqslant 0 .
\end{array}\right.
$$

So by Lemma $2.3, \lambda\left(\theta_{1}, f\right) \geqslant \lambda\left(\theta_{2}, f\right)$.

THEOREM 3.2 Let $f_{1}, f_{2} \in \mathscr{F}$ and let $(H \lambda)$ be satisfied both for $A=A^{f_{1}}, A^{f_{2}}$. Suppose further

$$
\begin{aligned}
& f_{1}^{\prime}(u)<f_{2}^{\prime}(u), \quad \text { on }[\theta, 1], \\
& f_{1}^{\prime \prime}(u) \leqslant 0, \quad \text { on }[\theta, 1],
\end{aligned}
$$

then

$$
\lambda\left(\theta, f_{1}\right) \geqslant \lambda\left(\theta, f_{2}\right) .
$$

Proof. From $(H f 1)$ and the fact that $f_{i}(1)=0, i=1,2$, it follows that $f_{1}(u) \geqslant f_{2}(u)$ on $[\theta, 1]$. Further, by defining

$$
F_{i}(u)=\int_{0}^{u} f_{i}(v) d v
$$

[see (1.8)] this inequality implies

$$
F_{1}(1)-F_{1}(u) \geqslant F_{2}(1)-F_{2}(u) \quad \text { on } \quad[\theta, 1]
$$

and (3.3) together with (1.8) gives for the respective solutions $V_{\theta, 1}, V_{\theta, 2}$ for $f=f_{1}, f_{2}$ of (1.7)

$$
V_{\theta, 1}^{\prime}(x) \geqslant V_{\theta, 2}^{\prime}(y), \quad \text { if } \quad V_{\theta, 1}(x)=V_{\theta, 2}(y) .
$$

Now define the function $w(x)$ as

$$
w(x)=l^{\theta, f_{2}}(x+\varepsilon),
$$

where $\varepsilon$ is a positive number to be specified later. By the positivity of the eigenfunction $l$ (Theorem 2.2) $w(0)>0$, and evaluation of $N_{1}[w]$ gives

$$
\begin{aligned}
N_{1}[w] & =-w^{\prime \prime}-f_{1}^{\prime}\left(V_{\theta, 1}(x)\right) w \\
& =\lambda\left(f_{2}\right) w+\left\{f_{2}^{\prime}\left(V_{\theta, 2}(x+\varepsilon)\right)-f_{1}^{\prime}\left(V_{\theta, 1}(x)\right)\right\} w \\
\geqslant & \lambda\left(f_{2}\right) w+\left\{f_{1}^{\prime}\left(V_{\theta, 2}(x+\varepsilon)\right)+\delta-f_{1}^{\prime}\left(V_{\theta, 1}(x)\right)\right\} w,
\end{aligned}
$$

where $\delta=\min \left\{f_{2}^{\prime}(u)-f_{1}^{\prime}(u) \mid \theta \leqslant u \leqslant 1\right\}$. By $(H f 1) \delta>0$ holds. Next, we define $\bar{x}=\bar{x}(\varepsilon)$ as the unique solution of $\left.V_{\theta, 2}(\bar{x}(\varepsilon)+\varepsilon)\right)=V_{\theta, 1}(\bar{x}(\varepsilon))$. The uniqueness follows from (3.4). We remark that $\bar{x}(\varepsilon) \downarrow 0$ for $\varepsilon \downarrow 0$. By (3.4) we have $V_{\theta, 1}(x) \geqslant V_{\theta, 2}(x+\varepsilon)$ for $x \geqslant \bar{x}$. This result, applied to (3.6) gives, using (Hf2),

$$
N_{1}[w] \geqslant \lambda\left(f_{2}\right) w, \quad x \in[\bar{x}, \infty) .
$$

Now choose $\varepsilon$ so small that

$$
f_{1}^{\prime}\left(V_{\theta, 1}(\bar{x}(\varepsilon))\right)+\delta \geqslant f_{1}^{\prime}\left(V_{\theta, 1}(0)\right)=f_{1}^{\prime}(\theta),
$$


then for $0 \leqslant x \leqslant \bar{x}$

$$
\begin{aligned}
& f_{1}^{\prime}\left(V_{\theta, 2}(x+\varepsilon)\right)+\delta \geqslant f_{1}^{\prime}\left(V_{\theta, 2}(\bar{x}+\varepsilon)\right)+\delta \\
= & f_{1}^{\prime}\left(V_{\theta, 1}(\bar{x})\right)+\delta \geqslant f_{1}^{\prime}\left(V_{\theta, 1}(0)\right) \geqslant f_{1}^{\prime}\left(V_{\theta, 1}(x)\right) .
\end{aligned}
$$

Using (3.8) and (3.6) we also find

$$
N_{1}[w] \geqslant \lambda\left(f_{2}\right) w, \quad x \in[0, \bar{x}] .
$$

So by Lemma $2.3, \lambda\left(\theta, f_{1}\right) \geqslant \lambda\left(\theta, f_{2}\right)$.

Corollary 3.1 Consider $f_{a,}, i=1,2 \in \mathscr{F}_{1}$ as given by (1.4). Suppose $(H \lambda)$ is satisfied for $f=f_{a_{i}}, i=1,2$, then Theorem 3.2 applies if $a_{i}<a_{2}$ and $\theta>\frac{1}{2}$.

Proof. Explicit calculation of condition (Hf2) requires $\theta \geqslant\left(1+a_{1}\right) / 3$, but condition (Hfl) requires more, namely $\theta>\frac{1}{2}$ and $a_{1}<a_{2}$.

Corollary 3.2 Consider $\tilde{f}_{v}, i=1,2 \in \mathscr{F}_{2}$ as given by (1.5). Suppose $(H \lambda)$ is satisfied for $f=\tilde{f}_{v_{\mathrm{i}}}, i=1,2$, then Theorem 3.2 applies if $v_{1}>v_{2}$ and $\theta>\frac{2}{3}$.

Proof. Condition $(H f 2)$ is satisfied for $\theta \geqslant 0$ if $-\frac{1}{2} \leqslant v_{1} \leqslant 1$ and $\theta \geqslant\left(v_{1}-1\right) /\left(3 v_{1}\right)$ if $v_{1} \geqslant 1$, but condition $(H f 1)$ requires more, namely $\theta>\frac{2}{3}$ and $v_{1}>v_{2}$.

\section{Positive Lower Bounds for $\lambda(\theta, f)$}

It was shown in Theorem 2.2 that $\lambda_{1}$, whenever it exists, is positive. In this section we shall show how, at the expense of additional conditions on $f$ and $\theta$, positive lower bounds can be found.

THEOREM 4.1 Let $(H \lambda)$ be satisfied, then

where

$$
\lambda(\theta, f) \geqslant \min \{J(u) \mid \theta \leqslant u \leqslant 1\}^{2},
$$

(see (1.9)).

$$
J(u)=f(u) / \sqrt{2(F(1)-F(u))} \text { on }[0,1) \text { and } J(1)=b=\sqrt{-f^{\prime}(1)}
$$

Remark. Because $J(u) \rightarrow b$, as $u \uparrow 1$ and $F(1)>F(u)$, on $(0,1), f \in C^{3}([0,1])$ for $f \in \mathscr{F}$ we have $J \in C^{2}([0,1])$ and bounded away from 0 on any interval $[\theta, 1]$ provided $\theta \in(a, 1)\left(a=0\right.$ if $\left.f \in \mathscr{F}_{2}\right)$. Thus for $f \in \mathscr{F}_{1}$ and $0 \leqslant \theta \leqslant a$, Theorem 4.1 does not give an improvement over the estimate $\lambda_{1}>0$.

Proof. Set $k=\min \{J(u) \mid \theta \leqslant u \leqslant 1\}$. We observe that in view of (1.7) and (1.8) $k=\min \left\{-V_{\theta}^{\prime \prime}(x) / V_{\theta}^{\prime}(x) \mid x \geqslant 0\right\}$. Now define $w(x)=e^{k x} V_{\theta}^{\prime}(x)$, then

$$
N[w]=w\left\{-k^{2}-2 k V_{\theta}^{\prime \prime} / V_{\theta}^{\prime}\right\}
$$

but since $J\left(V_{\theta}\right)=-V_{\theta}^{\prime \prime} / V_{\theta}^{\prime}$, we find

$$
N[w]=w\left\{-k^{2}+2 k J\left(V_{\theta}\right)\right\} \geqslant w\left\{-k^{2}+2 k^{2}\right\}=k^{2} w,
$$

from which by Lemma 2.3 the result follows.

Corollary 4.1 Let $(H \lambda)$ be satisfied and suppose $J^{\prime}(u) \geqslant 0$ on $[\theta, 1]$, then

$$
\lambda(\theta, f) \geqslant f^{2}(\theta) /\{2(F(1)-F(\theta))\} .
$$

Proof. From the extra condition on $J$ it follows that $J$ is non-decreasing on $[\theta, 1]$, so the minimum $k$ is found for $u=\theta$. 
Corollary 4.2 Let $(H \lambda)$ be satisfied and suppose $f^{\prime \prime}(u) \leqslant 0$ on $[\theta, 1]$, the estimate (4.2) follows.

Proof. Suppose there exists a number $u_{1}, \theta \leqslant u_{1}<1$ such that $J^{\prime}\left(u_{1}\right)<0$. Calculation gives $J^{\prime}=\left(f^{\prime}+J^{2}\right) / G$ and $J^{\prime \prime}=\left(f^{\prime \prime}+J J^{\prime}\right) / G$, where

$$
G(u)=\sqrt{2(F(1)-F(u))},
$$

which means that also $J^{\prime \prime}\left(u_{1}\right)<0$. But this fact implies that $J^{\prime}(u)<0$ on $\left[u_{1}, 1\right]$. However, explicit calculation of $J^{\prime}(1)$ reveals

$$
J^{\prime}(1)=-f^{\prime \prime}(1) /\left(3 \sqrt{-f^{\prime}(1)}\right)>0,
$$

which gives a contradiction. So $J^{\prime}\left(u_{1}\right) \geqslant 0$ on $[\theta, 1]$ and hence Corollary 4.1 applies.

Corollary 4.3 Let $(H \lambda)$ be satisfied and suppose for some $c, \theta<c<1, f^{\prime \prime}(u) \leqslant 0$ on $[c, 1]$ and $J^{\prime}(u) \geqslant 0$ on $[\theta, c]$, then estimate (4.2) follows.

Proof. Combine the two former corollaries.

Corollary 4.4 Consider $f_{a} \in \mathscr{F}_{1}$ as given by (1.4). Suppose $(H \lambda)$ is satisfied, then estimate (4.2) holds if $\theta>a$.

Proof. Apply Corollary 4.2 with $c=(1+a) / 3$ if $\theta>c$. Remark that $f^{\prime \prime}(c)=0$ and $f^{\prime \prime}(u) \leqslant 0$ on $[c, 1]$. Apply Corollary 4.3 if $a<\theta \leqslant c$. Because $J^{\prime}=\left(f^{\prime}+J^{2}\right) / G$ and $f^{\prime}>0$ on $\left(d_{1}, d_{2}\right)$ with $d_{1}<a<c<d_{2}$, where

$$
d_{1,2}=\left\{1+a \pm \sqrt{1-a+a^{2}}\right\} / 3
$$

represent the zeros of $f_{a}^{\prime}$, we find $J^{\prime}>0$ on $\left(d_{1}, d_{2}\right) \supset[\theta, c]$.

Corollary 4.5 Consider $\tilde{f}_{v} \in \mathscr{F}_{2}$ as given by (1.5). Suppose $(H \lambda)$ is satisfied, then estimate (4.2) holds if $\theta>0, v>-\frac{1}{2}$.

Proof. Apply Corollary 4.2 for $-\frac{1}{2} \leqslant v \leqslant 1$ and Corollary 4.3 for $v>1$ with $c=(v-1) /(3 v)$. Note $f^{\prime \prime}(c)=0$.

Next we give another estimate in which an integral norm is involved.

THEOREM 4.2 Let $(H \lambda)$ be satisfied, then

where

$$
\lambda(\theta, f) \geqslant-f^{\prime}(1)-c_{p}\|q\|_{p}^{(2 p) /(2 p-1)}, \quad p \geqslant 1,
$$

and

$$
q(x)=f^{\prime}(1)-f^{\prime}\left(V_{\theta}(x)\right), \quad\|q\|_{p}^{p}=\int_{0}^{\infty}|q|^{p} d x
$$

$$
\left.\begin{array}{l}
c_{1}=\frac{1}{4}, \\
c_{p}=p^{-(2 p) /(2 p-1)}(p-1)^{(2 p-2) /(2 p-1)}\left[\frac{\Gamma\left(\frac{1}{2}\right) \Gamma(p)}{\Gamma\left(p+\frac{1}{2}\right)}\right]^{-2 /(2 p-1)}, \quad p>1 .
\end{array}\right\}
$$

Proof. See Veling (1982b). Of course (4.3) gives only new information if the righthand side is positive.

Remark. For $p=1,2, \infty$ (4.3) gives respectively

$$
\lambda(\theta, f) \geqslant-f^{\prime}(1)-\frac{1}{4}\|q\|_{1}^{2},
$$




$$
\begin{aligned}
& \lambda(\theta, f) \geqslant-f^{\prime}(1)-\left(\frac{3}{16}\right)^{2 / 3}\|q\|_{2}^{4 / 3}, \\
& \lambda(\theta, f) \geqslant-f^{\prime}(1)-\sup _{x \geqslant 0}|q(x)| .
\end{aligned}
$$

In Section 7 calculations of estimates (4.2) and (4.3) for $p=2$ and $f=f_{a} \in \mathscr{F}_{1}$ are compared. Also the best possible result of (4.3) has been given by varying $p$ with steps of $\frac{1}{10}$ in the range $[1,3]$.

\section{Explicit Solution of the Eigenvalue Problem for $f(u)=u(1-u)$}

In this section the eigenvalue problem $A l=\lambda l$ for $f(u)=u(1-u)$ will be solved explicitly. First we gather some information about $f$ and the solution $V_{\theta}$ of (1.7). It turns out to be appropriate to express the functions in terms of the variable $z=1-u$.

$$
\begin{gathered}
\left.f(1-z)=z(1-z) ; \quad \begin{array}{c}
f^{\prime}(1-z)=-1+2 z ; \\
f^{\prime \prime}(1-z)=-2 ; \quad 2[F(1)-F(1-z)]=z^{2}\left(1-\frac{2}{3} z\right),
\end{array}\right\} \\
V_{\theta}(x)=1-\frac{3}{1+\cosh (x+A)}, \quad x \geqslant 0, \quad A=\operatorname{arccosh}\left(\frac{2+\theta}{1-\theta}\right) .
\end{gathered}
$$

In the foregoing sections we had put the natural restriction $0 \leqslant \theta \leqslant 1$. Extending the domain of $V_{\theta}$ to $\mathbb{R}$, we note that the range of $V_{\theta}$ is $\left[-\frac{1}{2}, 1\right]$. Hence we allow $\theta$ to lie in $\left[-\frac{1}{2}, 1\right]$. By the monotonicity of the transformation $z=1-V_{\theta}(x)$ it is possible to write $(1.10)$ as

$$
-2(F(1)-F(1-z)) v^{\prime \prime}-f(1-z) v^{\prime}-f^{\prime}(1-z) v=\lambda v, \quad 0 \leqslant z \leqslant 1-\theta, \quad '=\frac{d}{d z},
$$

where we have written $v(z)=w(x)$. Putting $\lambda=1-\rho^{2}$ and inserting (5.1) yields

$$
-z\left(1-\frac{2}{3} z\right) v^{\prime \prime}-(1-z) v^{\prime}+\left(\rho^{2}-2 z\right) v=0, \quad 0 \leqslant z \leqslant 1-\theta .
$$

Equation (5.4) represents a hypergeometric differential equation with regular singularities at $z=0, \frac{3}{2}, \infty$ and can be characterized with the aid of the Riemann's $P$-symbol (Abramowitz \& Stegun, 1965, 15.6.1, 15.6.3) as

$$
v(z)=P\left\{\begin{array}{cccc}
0 & 1 & \infty \\
\rho & 0 & 2 & \frac{2}{3} z \\
-\rho & \frac{1}{2} & -\frac{3}{2}
\end{array}\right\} .
$$

By Abramowitz \& Stegun $(1965,15.6 .11,15.6 .5,15.1 .1)(5.5)$ can be written as a multiple of

$$
\begin{aligned}
v(z) & =z^{\rho} P\left\{\begin{array}{ccc}
0 & 1 & \infty \\
0 & 0 & \rho+2 \\
-2 \rho & \frac{1}{2} & \rho-\frac{3}{2}
\end{array}\right\}=z^{\rho}{ }_{2} F_{1}\left(\rho+2, \rho-\frac{3}{2} ; 2 \rho+1 ; \frac{2}{3} z\right), \\
& =z^{\rho} \sum_{n=0}^{\infty} \frac{(\rho+2)_{n}\left(\rho-\frac{3}{2}\right)_{n}}{(2 \rho+1)_{n} n !}\left(\frac{2}{3} z\right)^{n},
\end{aligned}
$$

where $(a)_{n}=\Gamma(a+n) / \Gamma(a)$. The series is absolutely convergent for $|z| \leqslant \frac{3}{2}$. We remark 
that for $\rho=1$, which implies $\lambda=0, v(z)$ equals, by Abramowitz \& Stegun (1965, 15.1.8),

$$
v(z)=z_{2} F_{1}\left(3,-\frac{1}{2} ; 3 ; \frac{2}{3} z\right)=z \sqrt{1-\frac{2}{3} z}=\sqrt{2[F(1)-F(1-z)]},
$$

thus by $(1.8) v(z(x))=V_{\theta}^{\prime}(x)$ satisfies $N\left[V_{\theta}^{\prime}\right]=0$. This fact follows easily by differentiation of (1.7).

It is possible to obtain more information from (5.6). The value of the parameters of the ${ }_{2} F_{1}$-function are such that there exists a quadratic transformation: $v(z)$ can be written by Abramowitz \& Stegun $(1965,15.4 .13)$ in terms of a so-called associated Legendre function of the first kind $P_{v}^{\mu}$

$$
v(z)=z^{\rho} 2^{2 \rho} \Gamma(1+2 \rho)\left(\frac{2}{3} z\right)^{-\rho} P_{3}^{-2 \rho}\left(\sqrt{1-\frac{2}{3} z}\right),
$$

which in turn can be written, using Abramowitz \& Stegun (1965, 8.1.2)

$$
v(z)=3^{\rho}\left(\frac{1+\sqrt{1-\frac{2}{3} z}}{1-\sqrt{1-\frac{2}{3} z}}\right)^{-\rho}{ }_{2} F_{1}\left(-3,4 ; 1+2 \rho ;\left(1-\sqrt{1-\frac{2}{3} z}\right) / 2\right) .
$$

In fact this ${ }_{2} F_{1}$-function is a polynomial (Abramowitz \& Stegun, 1965, 15.4.1) in $r(x)$. Since we can write by $z=1-V_{\theta}(x)$ and (5.2)

$$
\begin{gathered}
\left(\frac{1+\sqrt{1-\frac{2}{3} z}}{1-\sqrt{1-\frac{2}{3} z}}\right)^{-\rho}=e^{-\rho(x+A),} \\
r(x)=\left(1-\sqrt{1-\frac{2}{3} z(x)}\right) / 2=(1-\operatorname{tgh}((x+A) / 2)) / 2,
\end{gathered}
$$

the representation of $w(x)$ becomes

$$
w(x)=v(z(x))=3^{\rho} e^{-\rho(x+A)} \sum_{n=0}^{3} \frac{(-3)_{n}(4)_{n}}{(1+2 \rho)_{n} n !}[(1-\operatorname{tgh}((x+A) / 2)) / 2]^{n} .
$$

Next we choose $\rho$ so that $w$ is an eigenfunction $(w(0)=0)$. Using (5.9) we require then

$$
{ }_{2} F_{1}(-3,4 ; 1+2 \rho ;(1-\sqrt{(1+2 \theta) / 3}) / 2)=0 .
$$

After some calculation and by (5.12) this equation becomes

$$
Q(\rho)=\rho^{3}+3 \sqrt{(1+2 \theta) / 3} \rho^{2}+((1+10 \theta) / 4) \rho+(5 \theta-2) \sqrt{(1+2 \theta) / 3} / 4=0 .
$$

Thus the eigenvalue problem $A l=\lambda l$ has been reduced to an algebraic one: to find a zero $\rho \in(0,1]$ of the cubic $Q$. The value $\rho=0$ is excluded since in that case $w \notin \mathscr{L}^{2}(0, \infty)$. A further analysis of $Q(\rho)=0$ reveals that for $-\frac{1}{2} \leqslant \theta<\frac{2}{5}$ there exists a unique monotonely decreasing solution $\rho \in(0,1]$ for increasing $\theta$ (see Theorem 3.1). For this range of $\theta$ there exists thus just one point $\lambda_{1}=\lambda(\theta) \in P \sigma\left(A^{\theta}\right)$. For $\frac{2}{5} \leqslant \theta \leqslant 1$ there is no solution and so $P \sigma\left(A^{\theta}\right)=\emptyset$.

We collect the results of this section in the next theorem.

THEOREM 5.1 The eigenfunction $l(x)$ of $A^{\theta} l=\lambda l$ when $f(u)=u(1-u)$ and $V_{\theta}(x)$ is given by (5.2) is represented by (5.12) where $\rho=\rho(\theta)$ is the unique solution of the equation $Q(\rho)=0$, in which $Q$ is given by (5.14) and $\rho \in(0,1], \theta \in\left[-\frac{1}{2}, \frac{2}{5}\right)$. The eigenvalue then equals $\lambda(\theta)=1-\rho^{2}(\theta)$. For $\theta \in\left[\frac{2}{5}, 1\right]$ there does not exist a solution of $A^{\theta} l=\lambda l$ and so $\operatorname{P} \sigma\left(A^{\theta}\right)=\emptyset$. 
We remark finally that the condition on $\theta$ which insures that $P \sigma\left(A^{\theta}\right)=\emptyset[$ (ii) in Lemma 2.1] can be evaluated quite easily. One finds

$$
\frac{2}{\pi} \int_{0}^{\infty} \sqrt{\left|f^{\prime}(1)-f^{\prime}\left(V_{\theta}(x)\right)\right|} d x=\frac{2 \sqrt{2}}{\pi} \int_{0}^{1-\theta} \frac{1}{\sqrt{z-\frac{2}{3} 2^{2}}} d z=\frac{4 \sqrt{3}}{\pi} \arcsin (\sqrt{2(1-\theta) / 3}),
$$

and so from Lemma 2.1 we learn that

$$
\theta>\theta_{0}=1-\frac{3}{2} \sin ^{2}\left(\frac{\pi}{4 \sqrt{3}}\right)=0.712 \Rightarrow P \sigma\left(A^{\theta}\right)=\emptyset .
$$

This is in agreement with Theorem 5.1, where $\theta_{0}$, has to be compared with the exact value $\frac{2}{5}$.

\section{The Eigenvalue Problem for $f(u)=u(1-u)(u-a)$}

In this section the eigenvalue problem $A l=\lambda l$ for the cubic non-linearity $f(u)=u(1-u)(u-a)$ will be studied. Once again we gather some information about $f$ expressed in the variable $z=1-u$ and the solution $V_{\theta}$ of (1.7)

$$
\begin{aligned}
& f(1-z)=z\left\{(1-a)-(2-a) z+z^{2}\right\}, \\
& f^{\prime}(1-z)=\left\{-(1-a)+2(2-a) z-3 z^{2}\right\} \\
& f^{\prime \prime}(1-z)=\{-2(2-a)+6 z\}, \\
& \left.2[F(1)-F(1-z)]=z^{2}\left\{(1-a)-\frac{2}{3}(2-a) z+\frac{1}{2} z^{2}\right\},\right\} \\
& \left.\begin{array}{c}
V_{\theta}(x)=1-\frac{6(1-a)}{2(2-a)+\sqrt{2-2 a-4 a^{2}} \sinh (\sqrt{1-a} x+B)}, \quad x \geqslant 0, \\
B=\operatorname{arcsinh}\left[\frac{\sqrt{2}[3-3 a-(1-\theta)(2-a)]}{(1-\theta) \sqrt{1-a-2 a^{2}}}\right] .
\end{array}\right\}
\end{aligned}
$$

In the same way as in Section 5 it is possible to rewrite (1.10) for the function $v(z)=w(x)$, with $z=1-V_{\theta}(x)$. Putting $\lambda=(1-a)\left(1-\rho^{2}\right)$ and using (6.1) we find

$$
\left.\begin{array}{c}
-v^{\prime \prime}+P(z) v^{\prime}+Q(z) v=0, \quad 0 \leqslant z \leqslant 1-\theta, \\
P(z)=\frac{1}{z}+\frac{\frac{1}{2}}{z-z_{1}}+\frac{\frac{1}{2}}{z-z_{2}}, \\
Q(z)=\frac{1}{z\left(z-z_{1}\right)\left(z-z_{2}\right)}\left[-6 z+4(2-a)-\frac{2(1-a) \rho^{2}}{z}\right] .
\end{array}\right\}
$$

where $z_{1}, z_{2}$ represent the zeros of $1-a-\frac{2}{3}(2-a) z+\frac{1}{2} z^{2}$ :

$$
z_{1,2}=\left[2(2-a) \pm i \sqrt{2-2 a-4 a^{2}}\right] / 3 .
$$

Equation (6.3) represents a differential equation with four regular singularities $\left(z=0, z_{1}, z_{2}, \infty\right)$ and Riemann's $P$-symbol is given by

$$
v(z)=P\left\{\begin{array}{cccc}
0 & z_{1} & z_{2} & \infty \\
\alpha_{0}=\rho & \alpha_{1}=0 & \alpha_{2}=0 & \alpha=3 \\
\beta_{0}=-\rho & \beta_{1}=\frac{1}{2} & \beta_{2}=\frac{1}{2} & \beta=-2
\end{array}\right\} .
$$


The parameters $\alpha, \beta, \alpha_{i}, \beta_{i}, i=1,2,3$ are found by the identification [see Snow, 1952, Ch. VII (1)]

$$
\begin{gathered}
P(z)=\frac{1-\alpha_{0}-\beta_{0}}{z}+\frac{1-\alpha_{1}-\beta_{1}}{z-z_{1}}+\frac{1-\alpha_{2}-\beta_{2}}{z-z_{2}}, \\
Q(z)=\frac{1}{\psi(z)}\left(\alpha \beta z+p+\alpha_{0} \beta_{0} \frac{\psi^{\prime}(0)}{z}+\alpha_{1} \beta_{1} \frac{\psi^{\prime}\left(z_{1}\right)}{z-z_{1}}+\alpha_{2} \beta_{2} \frac{\psi^{\prime}\left(z_{2}\right)}{z-z_{2}}\right), \\
\psi(z)=z\left(z-z_{1}\right)\left(z-z_{2}\right) .
\end{gathered}
$$

The so-called accessory parameter $p$ needs to be given as well and here equals

$$
p=4(2-a) \text {. }
$$

By the transformation $\bar{z}=z / z_{1}, v(z)=\bar{v}(\bar{z})=\bar{z}^{\rho} F(\bar{z})$ can be written as [see Snow, 1952, Ch. VII (2), (3), (4)]

with

$$
\bar{v}(\bar{z})=\bar{z}^{\rho} P\left\{\begin{array}{cccc}
0 & 1 & \bar{a}=\frac{z_{1}}{z_{2}} & \infty \\
0 & 0 & 0 & \bar{\alpha}=3+\rho \quad \bar{z} \\
1-\bar{\gamma}=-2 \rho & \bar{\gamma}+\delta-\bar{\alpha}-\bar{\beta}=\frac{1}{2} & 1-\bar{\delta}=\frac{1}{2} & \bar{\beta}=-2+\rho
\end{array}\right\},
$$

$$
\bar{p}=4(2-a)\left(\frac{3}{2}-\rho\right)(\rho+2) /\left(3 z_{1}\right) \text {. }
$$

The Riemann's $P$-symbol in the form (6.9) solves the differential equation

$$
F^{\prime \prime}+\left\{\frac{1-\bar{\gamma}}{\bar{z}}+\frac{\bar{\gamma}+\bar{\delta}-\bar{\alpha}-\bar{\beta}}{\bar{z}-1}+\frac{1-\bar{\delta}}{\bar{z}-\bar{a}}\right\} F^{\prime}+\left\{\frac{\bar{\alpha} \bar{\beta} \bar{z}+\bar{p}}{\overline{\bar{z}}(\bar{z}-1)(\bar{z}-\bar{a})}\right\} F=0 .
$$

The solution of (6.10) which is regular in the neighbourhood of $\bar{z}=0$ and belongs to the exponent zero is the Heun function denoted by [Snow, 1952, Ch. VII (6a), (7), $\left.\left(7^{\prime}\right)\right]$

$$
F(\bar{a}, \bar{p} ; \bar{\alpha}, \bar{\beta}, \bar{\gamma}, \delta ; \bar{z})=1-\frac{\bar{p}}{\bar{\gamma} \bar{\alpha}} \bar{z}+\sum_{n=2}^{\infty} c_{n} \bar{z}^{n},
$$

and the coefficients satisfy

$$
\left.\begin{array}{c}
c_{0}=1, \quad c_{1}=-\bar{p} /(\bar{\gamma} \bar{a}), \\
(n+2)(n+1+\bar{\gamma}) \bar{a} c_{n+2} \\
=\left\{(n+1)^{2}(\bar{a}+1)+(n+1)[\bar{\gamma}+\bar{\delta}-1+(\bar{\alpha}+\bar{\beta}-\bar{\delta}) \bar{a}]-\bar{p}\right\} c_{n+1}-(n+\bar{\alpha})(n+\bar{\beta}) c_{n} .
\end{array}\right\}
$$

So we find that, deleting insignificant factors,

$$
v(z)=z^{\rho} F\left(\frac{z_{2}}{z_{1}}, 4(2-a)\left(\frac{3}{2}-\rho\right)(\rho+2) /\left(3 z_{1}\right) ; 3+\rho,-2+\rho, 1+2 \rho, \frac{1}{2} ; \frac{z}{z_{1}}\right) .
$$

The recurrence relation becomes, defining $b_{n}=z_{1}^{-n} c_{n}$

$$
\left.\begin{array}{c}
b_{0}=1, \quad b_{1}=(2-a)(\rho+2)(2 \rho-3) /\{3(1-a)(2 \rho+1)\}, \\
b_{n}=\frac{1}{(1-a)(n+2 \rho) n}\left\{\frac{2}{3}(2-a)(n+\rho+1)\left(n+\rho-\frac{5}{2}\right) b_{n-1}-\right. \\
\left.\frac{1}{2}(n+\rho-4)(n+\rho+1) b_{n-2}\right\} .
\end{array}\right\} .
$$


We note that this recurrence relation is identical to the one which has been found by Greenberg (1981) if one changes $a$ into $1-a$. He studied the eigenvalue problem for the linearization of $u_{x x}+f(u)=0$ with respect to the function $W(x)$ satisfying

$$
\left.\begin{array}{c}
W^{\prime \prime}+W(1-W)(W-a)=0, \quad-\infty<x<\infty, \\
W(-\infty)=W(\infty)=0 .
\end{array}\right\}
$$

It is well known that the asymptotic behaviour of a recurrence relation

where

$$
b_{n}+a_{n} b_{n-1}+c_{n} b_{n-2}=0
$$

$$
\lim _{n \rightarrow \infty} a_{n}=A, \lim _{n \rightarrow \infty} c_{n}=C,
$$

can be found by determining the roots of $t^{2}+A t+C=0$ (see, e.g., Hunter, 1968). Here we find

where

$$
b_{n} \sim C_{0} R^{n} \cos (n \phi+r \log n), \quad n \rightarrow \infty,
$$

$R=\left|t_{1}\right|=\left|t_{2}\right|=1 / \sqrt{2-2 a}<1, \phi=\arctan \left(p h t_{1}\right)=\arctan \left(\sqrt{1-a-2 a^{2}} /[(2-a) \sqrt{2}]\right.$, $C_{0}$ is a constant determined by the initial values and $r$ is a constant which needs a higher-order asymptotic study.

By the knowledge of a possible candidate $l(x)$ of $A l=\lambda l$ we solve the eigenvalue problem by determining $\rho$ such that $v(1-\theta)=0$ which amounts to locating the zeros $\rho \in(0,1)$ of

$$
F\left(\frac{z_{2}}{z_{1}}, 4(2-a)\left(\frac{3}{2}-\rho\right)(\rho+2) /\left(3 z_{1}\right) ; 3+\rho,-2+\rho, 1+2 \rho, \frac{1}{2} ; \frac{1-\theta}{z_{1}}\right)=0 .
$$

The number of zeros is equal to the number of points in $P \sigma(A)$. The series representation (6.11) with (6.14) offers a suitable tool to perform these calculations numerically. In Section 7 we determined the eigenvalue $\lambda$ for different choices of $\theta$ and the zero $a$ of $f(u)$ by this technique.

We collect the result of this section in the next theorem.

THEOREM 6.1 The eigenfunction $l(x)$ of $A l=\lambda l$ for $f_{a}(u)=u(1-u)(u-a)$ and $V_{\theta}(x)$ given by (6.2) is represented, if it exists, by $l(x)=v\left(1-V_{\theta}(x)\right)$, where $v(z)$ is given in (6.13) and $\rho$ is a solution of $(6.17), \rho \in(0,1)$. The corresponding eigenvalue then equals $\lambda=(1-a)\left(1-\rho^{2}\right)$.

\section{Numerical Results}

As was announced in the previous sections the numerical calculations involving some of the equations will be summarized in this section. In all the calculations below we have taken the non-linearity

$$
f(u)=f_{a}(u)=u(1-u)(u-a) \in \mathscr{F}_{1}
$$

as an example. We have made three choices of $a(0 \cdot 1,0 \cdot 25,0 \cdot 4)$ and eleven choices of $\theta(0,0 \cdot 1,0 \cdot 2, \ldots, 0 \cdot 8,0 \cdot 9$, and $0 \cdot 95)$.

In Table 1 the results of calculations based on Lemmas 1 and 2 are shown. 
TABLE 1

$P \sigma(A)$

\begin{tabular}{lllllllllllll}
\hline$a \backslash \theta$ & 0 & 0.1 & 0.2 & 0.3 & 0.4 & 0.5 & 0.6 & 0.7 & 0.8 & 0.9 & 0.95 \\
\hline 0.1 & + & + & + & + & + & + & + & $\times$ & - & - & - \\
0.25 & + & + & + & + & + & + & + & + & $\times$ & - & - \\
0.4 & + & + & + & + & + & + & + & + & $\times$ & - & - \\
\hline
\end{tabular}

+: $P \sigma(A)$ is not empty according to Lemma 2.1 with $\rho(x)=e^{\gamma x}-1, \gamma>0$.

-: $P \sigma(A)$ is empty according to Lemma 2.2 , (i) for $p=1$.

$x$ : Both methods above failed to give information; calculation of $\bar{\lambda}_{1}=\left\|\rho V_{\theta}^{\prime}\right\|_{2}^{2} /\left\|\rho^{\prime} V_{\theta}^{\prime}\right\|_{2}^{2}$ revealed:

$a=0.1, \quad \theta=0.7$, for $\gamma=0.9050 \lambda_{1}=0.9035>0.9=-f_{a}^{\prime}(1)$,

$a=0.25, \theta=0.8$, for $\gamma=0.8400 \lambda_{1}=0.7605>0.75=-f_{a}^{\prime}(1)$,

$a=0.4, \quad \theta=0.8$, for $\gamma=0.7440 \lambda_{1}=0.6071>0.6=-f_{a}^{\prime}(1)$.

In Table 2 we show:

(A) The eigenvalues $\lambda_{1}$ calculated up to three significant digits by the method of Theorem 6.1. A finite-element method gave the same results up to the required precision. For the entry with the ' - ' symbol, there does not exist a solution of (6.17), so $\operatorname{P} \sigma(A)=\emptyset$. The same conclusion holds for $a=0.25$, $\theta=0.8$ and $a=0.4, \theta=0.8$ (compare Table 1 ). For the entry $a=0.4, \theta=0$ there exists a second eigenvalue $\lambda_{2}=0.598$.

(B) The lower bounds of Theorem 4.2 for $p=2$.

(C) By varying $p$ in the interval $[1,3]$ with stepsize $\frac{1}{10}$ it is possible to improve the bound under $(B)$.

TABLE 2

Eigenvalue $\lambda$ (see text)

\begin{tabular}{lccccccccc}
\hline$a \backslash \theta$ & & 0 & 0.1 & 0.2 & 0.3 & 0.4 & 0.5 & 0.6 & 0.7 \\
\hline 0.1 & (A) & 0.459 & 0.540 & 0.625 & 0.708 & 0.783 & 0.844 & 0.885 & - \\
& (B) & 0.206 & 0.2396 & 0.287 & 0.349 & 0.421 & 0.501 & 0.587 & \\
& (C) & 0.209 & 0.2397 & 0.288 & 0.354 & 0.433 & 0.523 & 0.618 & \\
& $p=$ & 2.2 & 2.1 & 1.9 & 1.8 & 1.7 & 1.6 & 1.5 & \\
& (D) & $\times$ & $\times$ & 0.0019 & 0.0139 & 0.0450 & 0.1021 & 0.1901 & \\
0.25 & (A) & 0.305 & 0.375 & 0.454 & 0.535 & 0.612 & 0.677 & 0.725 & 0.749 \\
& (B) & 0.135 & 0.156 & 0.192 & 0.243 & 0.306 & 0.378 & 0.456 & 0.539 \\
& (C) & 0.142 & 0.159 & 0.192 & 0.244 & 0.311 & 0.391 & 0.479 & 0.568 \\
& $p=$ & 2.3 & 2.2 & 2.0 & 1.9 & 1.8 & 1.6 & 1.5 & 1.4 \\
& (D) & $\times$ & $\times$ & $\times$ & 0.0012 & 0.0156 & 0.0535 & 0.1213 & 0.2229 \\
0.4 & (A) & 0.137 & 0.193 & 0.267 & 0.348 & 0.429 & 0.502 & 0.559 & 0.593 \\
& (B) & 0.0503 & 0.0606 & 0.0870 & 0.1292 & 0.184 & 0.250 & 0.323 & 0.400 \\
& (C) & 0.0669 & 0.0722 & 0.0914 & 0.1294 & 0.185 & 0.256 & 0.337 & 0.421 \\
& $p=$ & 2.6 & 2.5 & 2.3 & 2.1 & 1.9 & 1.7 & 1.6 & 1.4 \\
& (D) & $\times$ & $\times$ & $\times$ & $\times$ & $\times$ & 0.0130 & 0.0568 & 0.1356 \\
\hline
\end{tabular}


(D) The lower bound of Corollary 4.4. For the entries with the ' $x$ ' symbol, the bound given by (4.2) is not applicable.

It turns out that (D) is inferior to (C).

We remark that the results are in agreement with Theorem 3.1 (monotonicity in $\theta$ ) and Corollary 3.1 (monotonicity in $a$ ).

The author thanks M. Bakker and R. Montijn for performing some calculations for Tables 1 and 2. He would also like to express his gratitude to Prof. L. A. Peletier for reading the manuscript and suggesting many improvements.

\section{REFERENCES}

Abramowitz, M. \& Stegun, I. A. (Eds) 1965 Handbook of Mathematical Functions. New York: Dover Publications.

Adams, R. A. 1975 Sobolev Spaces. New York: Academic Press.

Aronson, D. G. \& Weinberger, H. F. 1975 Nonlinear diffusion in population genetics, combustion, and nerve pulse propagation. In Partial Differential Equations and Related Topics (Goldstein, J. A., Ed.), pp. 5-49. Proc. Tulane Program Partial Differential Equations. Lecture Notes in Mathematics 446. Berlin: Springer-Verlag.

Chaudhuri, J. \& Everitr, W. N. 1967 On the spectrum of ordinary second order differential operators. Proc. R. Soc. Edinb. A68, 95-119.

Dunford, N. \& Schwartz, J. T. 1963 Linear Operators, Part II. New York: Interscience.

EVERITT, W. N. 1972 On the spectrum of a second order linear differential equation with a $p$ integrable coefficient. Applicable Analysis, 2, 143-160.

FIFE, P. C. 1979 Mathematical Aspects of Reacting and Diffusing Systems. Lecture Notes in Biomathematics 28. Berlin: Springer-Verlag.

FIFE, P. C. \& MCLEOD, J. B. 1977 The approach to solutions of nonlinear diffusion equations to travelling front solutions. Archs ration. Mech. Analysis 65, 335-361.

FISHER, R. A. 1977 The wave of advance of advantageous genes. Ann. Eugenics 7, 335-369.

Greenberg, J. M. 1981 Stability of equilibrium solutions for the Fisher equation. Q. appl. Math. 39, 239-247.

Hunter, C. 1968 Asymptotic solutions of certain linear difference equations, with applications to some eigenvalue problems. J. Math. Analysis Applic. 24, 279-289.

NaImark, M. A. 1968 Linear Differential Operators, Part II (Dawson, E. R. \& Everitt, W. N., Trans.). New York: Frederick Ungar.

Protter, M. H. \& Weinberger, H. F. 1966 On the spectrum of general second order operators. Bull. Am. math. Soc. 72, 251-255.

Protter, M. H. \& Weinberger, H. F. 1967 Maximum Principles in Differential Equations. Englewood Cliffs, N.J.: Prentice-Hall.

Reed, M. \& Simon, B. 1978 Methods of Modern Mathematical Physics, Part IV. New York: Academic Press.

SNow, C. 1952 Hypergeometric and Legendre Functions with Applications to Integral Equations of Potential Theory. National Bureau of Standards, Applied Mathematics Series 19 , Washington.

Titchmarsh, E. C. 1962 Eigenfunctions Expansions Associated With Second-order Differential Equations, Part I. Oxford: Oxford University Press.

Veling, E. J. M. 1981 Travelling waves in an initial-boundary value problem. Proc. R. Soc. Edinb. A90, 41-61.

Veling, E. J. M. $1982 a$ Pushed travelling waves in an initial-boundary value problem for Fisher type equations. Nonlinear Analysis, Theory, Meth. Applics 6, 1271-1286.

VELING, E. J. M. $1982 b$ Optimal lower bounds for the spectrum of a second order linear differential equation with a $p$-integrable coefficient. Proc. R. Soc. Edinb. A92, 95-101. 